\section{Praxismanual Strahlentherapie}

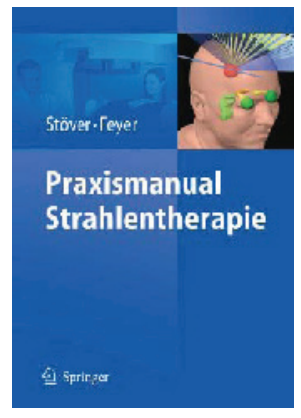

Das neue Buch

„Praxismanual

Strahlenthera-

pie" der Auto-

rinnen Stöver \&

Feyer ist ein Le-

xikon, welches

durch seine

Vollständigkeit

und Kompri-

miertheit besticht. Zu jedem onkologischen Thema und zu jeder Tumorentität, wie auch jeder Indikation bei gutartigen Erkrankungen, finden sich in tabellarischer Form immens viele Informationen, die die Autorinnen sehr gut recherchiert und zusammengestellt haben. Das Buch ist eine sinnvolle und wichtige Grundlage für die Kollegen der Strahlentherapie aber insbesondere auch für die Kollegen, die den Strahlentherapeuten zuweisen. Dieser kann sich in dem vollständigen Glossar und Stichwortverzeichnis schnell orientieren, inwieweit eine Strahlentherapie bei den gegebenen Erkrankungen in Frage kommt. Der Fachkollege wiederum findet in dem Buch eine sehr gute Grundlage, seine eigenen Indikationsansichten abzuklären und kann, nach meiner Meinung, einen Großteil der angegebenen Informationen direkt in sein eigenes Hauskonzept einbringen.

Ich empfehle das Werk wärmstens für alle Strahlentherapeuten und insbesondere auch für die Zuweiser anderer Fachrichtungen. Der Preis von 59.95 Euro ist für den guten Druck und die 430 Seiten gerechtfertigt.

G. Gademann (Magdeburg)

> Imke Stöver, Petra Feyer

Praxismanual Strahlentherapie

Heidelberg, Springer-Verlag GmbH 2011 435 S. (ISBN 978-3-642-10536-4), Soft

Cover, 59.95 EUR

\section{Hormonelle Situation}

Bei prämenopausalen Frauen und lymphonodal nichtmetastasierter Erkrankung und Plattenepithelkarzinom können die Adnexe erhalten werden. Ist eine primäre Radiochemotherapie notwendig, so kann eine Verlagerung der Adnexe aus dem Bestrahlungsgebiet erfolgen. Allerdings sind aufgrund der potenziellen Streustrahlung nur bei der Hälfte der Frauen dann die Ovarien endokrin wirksam. Da bei Vorliegen eines Adenokarzinoms bei bis zu 5\% der Patientinnen die Adnexe tumorbefallen sein können, wird bei der chirurgischen Therapie die Mitentfernung der Adnexe oder bei der primären Radiochemotherapie die Einbeziehung der Adnexe in das tumorbezogene Zielvolumen präferiert.

Ist bei Frauen mit Kinderwunsch aufgrund der Tumorausdehnung die primäre Radiochemotherapie notwendig, so kann vor Beginn eine Eizellgewinnung und Kryokonservierung erfolgen oder alternativ Ovarialgewebe entnommen und kryokonserviert werden. Das Paar muss dann allerdings über die Notwendigkeit einer Leihmutter (in Deutschland nicht erlaubt) aufgeklärt werden.

\section{Paraaortaler N1-Status}

Durch das laparoskopische Staging wird bei Frauen mit fortgeschrittener Tumorerkrankung auch der paraaortale Status evaluiert. Sind die pelvinen Lymphknoten befallen und die paraaortalen Lymphknoten histopathologisch tumorfrei, so kann auf die paraaortale Mitbestrahlung verzichtet werden. Sind die paraaortalen Lymphknoten befallen und entfernt worden, erfolgt eine Evaluierung des Skalenuslymphknotens. Auch bei isoliertem Befall der paraaortalen Lymphknoten und freien Skalenuslymphknoten besteht weiterhin ein kurativer Ansatz durch die primäre Radiochemotherapie.

\section{Tumorpersistenz nach primärer Radiochemotherapie}

Nach primärer Radiochemotherapie wird der lokale Status bezüglich Tumorfreiheit mittels bildgebender Verfahren und histopathologischer Evaluierung der ver- bliebenen Zervix evaluiert. Bei Tumorpersistenz kann eine Hysterektomie oder ggf. Exenteration notwendig werden. Bei komplett erfolgter Radiochemotherapie inkl. Brachytherapie ist aber im Regelfall keine Hysterektomie notwendig.

\section{Unterbehandeltes Zervixkarzinom}

Erfolgt durch Nichtkenntnis der Diagnose eine einfache Hysterektomie, dann ist als weitere Behandlung die laparoskopische Evaluierung des Lymphknotenstatus und bei tumorfreien Lymphknoten entweder die radikale laparoskopisch-vaginale Parametrektomie oder die Radiochemotherapie indiziert.

\section{Lokalrezidiv}

Tritt ein lokoregionäres Rezidiv auf, so wird bei Zustand nach primärer chirurgischer Behandlung die Radiochemotherapie durchgeführt. Geht primär eine Radiochemotherapie oder chirurgische Therapie mit folgender Radiochemotherapie voraus, so erfolgt die Exenteration mit einer 5-Jahres-Überlebensrate von ca. 40\%. Vor Exenteration ist die Durchführung einer Staginglaparoskopie sinnvoll, um der Patientin bei Kontraindikation für Exenteration wie intraabdominale Tumoraussaat oder der fehlenden Möglichkeit einer Ro-Resektion die Explorationslaparatomie zu ersparen.

\section{Metastasierung}

Bei primär metastasierter oder rezidivierter metastasierter Erkrankung beträgt die mediane Überlebenszeit zwischen 6 und 9 Monaten. Die Cisplatinmonotherapie zeigt Ansprechraten zwischen 20-30\%. Eine Kombinationstherapie aus Cisplatin und Topotecan bei rezidivierender Erkrankung ergab eine Verlängerung des medianen Überlebens durch die Kombinationstherapie von 6,5 auf 9,5 Monate. Daher gilt die Kombinationstherapie im Moment als Standard.

\section{Nachsorge}

Bei der Nachsorge erfolgt im Zeitabstand von 3 Monaten die symptombezogene Anamnese, körperliche Untersuchung, 